\title{
Study on the use of omega- 3 fatty acids as a therapeutic supplement in treatment of psoriasis
}

This article was published in the following Dove Press journal:

Clinical, Cosmetic and Investigational Dermatology

17 May 2011

Number of times this article has been viewed

\section{G Márquez Balbás \\ M Sánchez Regaña \\ $P$ Umbert Millet}

Psoriasis and Phototherapy Unit, Hospital Universitario Sagrat Cor, Barcelona, Spain
Correspondence: G Márquez Balbás Psoriasis and Phototherapy Unit, Hospital Universitario Sagrat Cor, Barcelona, Spain.

Email 4087Igmb@comb.cat

\begin{abstract}
Previous studies have suggested a benefit for patients with plaque psoriasis when omega-3 fatty acids are added to topical treatment. This study evaluated the efficacy of a nutritional complement rich in omega-3 fatty acids in patients with mild or moderate plaque psoriasis. Thirty patients were recruited, 15 of whom were given topical treatment with tacalcitol, forming the control group. The remaining 15 patients were given topical tacalcitol and 2 capsules of Oravex ${ }^{\circledR}$ daily. Three visits, the baseline, intermediate (week 4), and final (week 8), were held over an 8-week period. The main efficacy endpoints were the Psoriasis Area and Severity Index (PASI), Nail Psoriasis Severity Index (NAPSI) and Dermatological Life Quality Index (DLQI). A clear and significant improvement was observed in all the efficacy endpoints in both groups between the baseline visit and the end visit. This improvement was significantly greater in the group treated additionally with Oravex ${ }^{\circledR}$ than in the control group. Supplementary treatment with omega-3 fatty acids complements topical treatment in psoriasis, and makes a significant contribution to reducing PASI and NAPSI and improving DLQI; and to reducing scalp lesion and pruritus, erythema, scaling, and infiltration of the treated areas.
\end{abstract}

Keywords: psoriasis, metabolic syndrome, vitamin D derivates, omega-3 fatty acids, tacalcitol

\section{Introduction}

Psoriasis is a chronic skin disease which shows a course with outbreaks and presents variable clinical findings. ${ }^{1}$ Nowadays, psoriasis is regarded as an immunologically based disease which combines dermal inflammation with secondary epidermal hyperplasia. $^{2}$

In recent years, the so-called metabolic syndrome has been described, and defined as the ensemble of cardiovascular risk factors, including central obesity, atherogenic dyslipidemia, hypertension, and glucose intolerance, affected by lifestyle, genetic predisposition, and the environment. The presence of metabolic syndrome triples a patient's risk of developing type 2 diabetes mellitus and doubles the risk of developing cardiovascular disease. ${ }^{3}$

It has been described that patients diagnosed with psoriasis have a high prevalence of metabolic disorders such as diabetes, hypertension, obesity, and hyperlipidemia, as well as a high frequency of tobacco consumption, and also an increased morbidity and mortality risk. ${ }^{4-6}$

Like psoriasis, metabolic syndrome is characterized by a fundamentally TH1 inflammatory response, which points to the hypothesis that psoriasis is associated 
with metabolic syndrome due to a shared inflammation mechanism. Another possible explanation for predisposition to the development of metabolic syndrome by patients with psoriasis lies in behavioral habits or in the psychological impact of the disease. Another hypothesis is that it is the actual metabolic syndrome that promotes development of psoriasis.

In relation to the foregoing, several studies have demonstrated an increase in C-reactive protein in patients with psoriasis due to an elevation in the serum concentration of IL-6 and MRP8 and MRP14 proteins, which presents proatherogenic action. ${ }^{4}$ Recent studies confirm that Th17, Th22, and Th1 cells are detected in psoriatic skin lesions and implicated in psoriasis pathogenesis. ${ }^{7}$

In recent years, the topical treatment of psoriasis has made major progress. ${ }^{8-10}$ Vitamin D derivatives are one of the pillars of topical treatment of psoriasis. ${ }^{11-15}$ In Spain 3 vitamin D derivatives are used: calcipotriol, tacalcitol, and calcitriol. In combination with other treatments, vitamin D derivatives constitute the ideal maintenance treatment, since their application may be maintained over time.

Omega-3 fatty acids, eicosapentaenoic acid (EPA) and docosahexaenoic acid (DHA), reduce symptoms in many inflammatory skin diseases, limiting the spreading of the inflammatory process. ${ }^{16,17}$ In the skin, the main route for the synthesis of leukotrienes is 15-lipoxygenase, giving rise to 15-hydroxyeicosatetraenoic acid. Moreover, the epidermis can convert the leukotriene A4 (produced by the polymorphonuclear leukocytes) into leukotriene B4, one of the main inflammation mediators.

The intake of EPA and DHA leads to the formation of hydroxylated metabolites through 15-lipoxygenase, 15-hydroxyeicosapentaenoic acid, and 15-hydroxydocosahexaenoic acid. Both substances are potent inhibitors of the 5-lipoxygenase of mononuclear cells, thus limiting the synthesis of proinflammatory leukotrienes LTB4, LTC4, and LTD4.

To date, several studies have been performed to evaluate the efficacy of the daily supplementation of EPA and DHA in patients with psoriasis, ${ }^{17-20}$ with an improvement observed in their mean Psoriasis Area and Severity Index (PASI) score, as well as in clinical symptoms, particularly in pruritus.

\section{Objective}

The study endpoint is to evaluate the efficacy of the intake of omega-3 fatty acids in patients with mild or moderate plaque psoriasis by means of the addition of Oravex ${ }^{\circledR}$ (Thea
Laboratories, Barcelona, Spain [280 mg of eicosapentaenoic acid, $40 \mathrm{mg}$ of docosahexaenoic acid, $50 \mathrm{mg}$ of thyme extract, $50 \mathrm{mg}$ of olive leaf extract, $20 \mathrm{mg}$ of green tea extract, $7.5 \mathrm{mg}$ of zinc, $27.5 \mu \mathrm{g}$ of selenium per capsule]), as a nutritional complement rich in omega-3 fatty acids, increases the efficacy of topical treatment of plaque psoriasis.

\section{Methodology}

This was a prospective, open, single-center, controlled observational study with a 2-month follow-up.

Fifteen patients were selected during October and November 2007 and began treatment with tacalcitol, forming group B. In turn, a further 15 patients were selected who, besides treatment with tacalcitol, took 2 daily capsules of Oravex $^{\circledR}$, forming group A. There was no placebo in the study, so the study was not blinded to either the patient or to the observer. Both groups were homogenous for sex, age, and body mass index (BMI). The dosage of EPA and DHA was $640 \mathrm{mg}$ daily (the World Health Organization recommends a minimum daily intake of 0.3 to $0.5 \mathrm{~g}$ of EPA and DHA in the general population).

The inclusion criteria were age between 18 and 70 years, and stable mild (PASI < 3) or moderate (PASI 3-10) plaque psoriasis. The exclusion criteria were receiving topical therapy in the previous 2 weeks and phototerapy, systemic, or biological therapy in the previous 4 weeks. Also we excluded pregnant or breastfeeding women, and patients with liver disease and neuropathies.

The main efficacy endpoints were PASI, Nail Psoriasis Severity Index (NAPSI), and Dermatology Life Quality Index (DLQI).

Secondary endpoints were the values pertaining to the presence of pruritus (yes/no), scalp psoriasis ( 0 , no; 1 , mild; 2 , moderate; 3 , severe) and clinical evolution of the target plaque according to erythema, infiltration, and scaling (scored on a scale of 0 , none to 5 , serious).

Blood analyses were performed on the patients at the baseline and end visits.

\section{Results}

Data were collected from the 30 patients at the baseline visit and weeks 4 and 8 (Table 1). No patient dropped out or was withdrawn from the study.

The mean age was $58.97 \pm 15.1$ years, $63.3 \%$ were men, and mean BMI was $26.85 \pm 3.3 \mathrm{~kg} / \mathrm{m}^{2} ; 30.0 \%$ of the patients had a normal BMI, 53.3\% were overweight, and the remaining $16.7 \%$ were considered obese. 
Table I Results of primary and secondary endpoints

\begin{tabular}{|c|c|c|c|c|c|}
\hline & $\begin{array}{l}\text { Baseline } \\
\text { visit week } 0\end{array}$ & $\begin{array}{l}\text { Intermediate } \\
\text { visit week } 4\end{array}$ & $\begin{array}{l}\text { End visit } \\
\text { week } 8\end{array}$ & $\begin{array}{l}\text { Difference } \\
\text { baseline-end }\end{array}$ & $\begin{array}{l}\text { Significance } \\
\text { baseline-end }\end{array}$ \\
\hline \multicolumn{6}{|l|}{ PASI } \\
\hline Tacalcitol & 8.53 & 6.53 & 5 & -3.53 & $P<0.000 \mathrm{I}$ \\
\hline Tacalcitol + Oravex & 9.6 & 5.4 & 2.8 & -6.8 & \\
\hline \multicolumn{6}{|l|}{ NAPSI } \\
\hline Tacalcitol & 0.38 & 0.43 & 0.38 & 0 & $P=0.0480$ \\
\hline Tacalcitol + Oravex & 2.91 & 2.45 & 1.68 & -1.23 & \\
\hline \multicolumn{6}{|l|}{ DLQI } \\
\hline Tacalcitol & 5.67 & 3.67 & 2.64 & -3.03 & $P=0.0056$ \\
\hline Tacalcitol + Oravex & 8.53 & 2.63 & 1.86 & -6.67 & \\
\hline \multicolumn{6}{|l|}{ Scalp lesion } \\
\hline Tacalcitol & 0.6 & 0.53 & 0.6 & 0 & $P=0.0038$ \\
\hline Tacalcitol + Oravex & 0.87 & 0.8 & 0.27 & -0.6 & \\
\hline \multicolumn{6}{|l|}{ Pruritus } \\
\hline Tacalcitol & $66.7 \%$ & $40.0 \%$ & $26.7 \%$ & $-40 \%$ & \\
\hline Tacalcitol + Oravex & $80.0 \%$ & $6.7 \%$ & $0 \%$ & $-80 \%$ & $P<0.000 \mathrm{I}$ \\
\hline \multicolumn{6}{|c|}{ Lesion of target plaque } \\
\hline \multicolumn{6}{|c|}{ Erythema } \\
\hline Tacalcitol & 2.20 & 1.80 & 1.47 & -0.73 & $P=0.0080$ \\
\hline Tacalcitol + Oravex & 2.93 & 1.80 & 1.13 & -1.80 & \\
\hline \multicolumn{6}{|l|}{ Infiltration } \\
\hline Tacalcitol & 1.53 & 0.93 & 0.53 & -1.0 & $P=0.0011$ \\
\hline Tacalcitol + Oravex & 2.47 & 1.13 & 0.47 & -2.0 & \\
\hline \multicolumn{6}{|l|}{ Scaling } \\
\hline Tacalcitol & 1.60 & 0.87 & 0.20 & -1.40 & $P=0.0291$ \\
\hline Tacalcitol + Oravex & 2.27 & 1.00 & 0.13 & -2.14 & \\
\hline
\end{tabular}

The safety endpoints, particularly in blood control, presented no deterioration in patient state rendering withdrawal from the study advisable.

The PASI presented an improvement of 6.8 points in the Oravex ${ }^{\circledR}$ group, whereas in the control group it was only 3.5 points (Figure 1 ). This difference was highly significant $(P<0.0001)$.

A reduction was observed in the NAPSI in the group treated with Oravex ${ }^{\circledR}$, from 2.91 at the baseline visit to 1.68 in the visit after 8 weeks. The control group did not improve in this endpoint, although this endpoint was initially very low and different to that of group A (Figure 2).

DLQI was also greater in the group treated with Oravex ${ }^{\circledR}$, with an improvement of 6.67 points versus 3.03 in the control group (Figure 3).

In all the secondary endpoints studied (scalp lesion, lesion of target plaque-erythema, infiltration, and scaling) a significant improvement was observed in the group treated

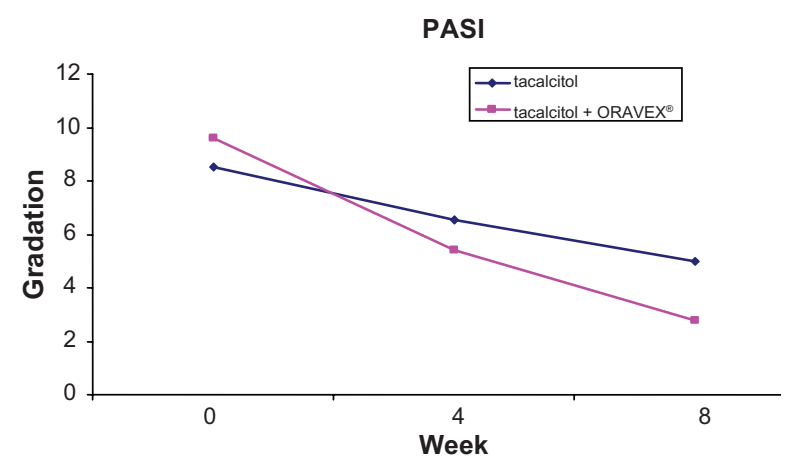

Figure I Evolution of psoriasis area and severity index in control group and Oravex group.

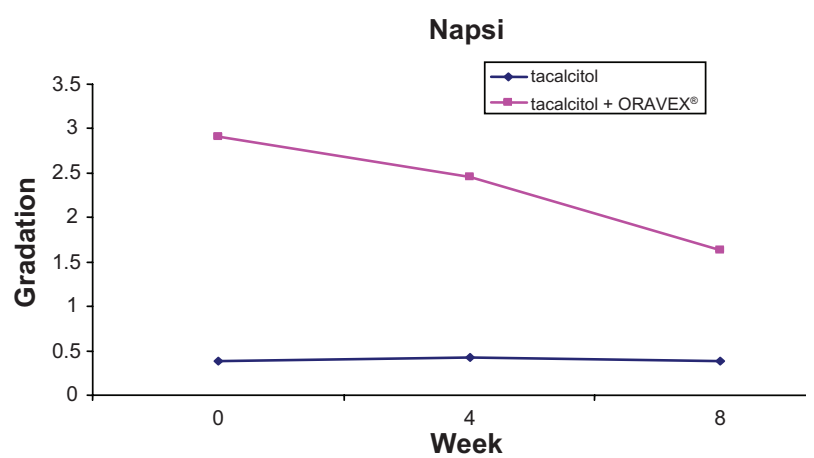

Figure 2 Evolution of nail psoriasis severity index in control group and Oravex ${ }^{\circledR}$ group. 


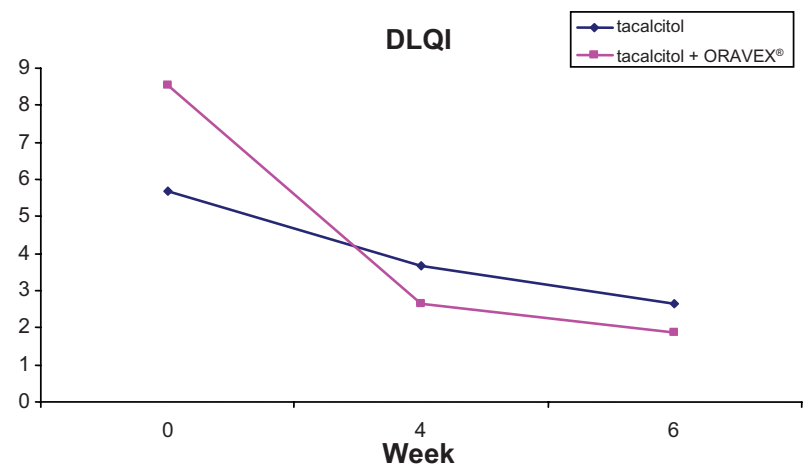

Figure 3 Evolution of dermatological life quality index in control group and Oravex ${ }^{\circledR}$ group.

with Oravex ${ }^{\circledR}$ compared with the control group after 8 weeks of treatment.

The evolution of some of the patients of the group treated with Oravex ${ }^{\circledR}$ plus tacalcitol is shown in Figures 4 and 5 .

\section{Discussion}

The present study was conducted to evaluate the efficacy of the addition of a nutritional supplement rich in omega-3 fatty acids in the treatment of psoriasis. Previous studies have demonstrated the possible effect of the diet on the evolution of the disease. Dietary omega-3 (n-3) fatty acids have a variety of anti-inflammatory and immune-modulating effects that may be of relevance to atherosclerosis and its clinical manifestations of myocardial infarction, sudden death, and stroke. A variety of biologic effects of EPA and DHA have been demonstrated from feeding studies with fish or fish oil supplements in humans and animals. These include effects on triglycerides, high-density lipoprotein cholesterol, platelet function, endothelial and vascular function, blood pressure, cardiac excitability, measures of oxidative stress, pro- and anti-inflammatory cytokines, and immune function. Clinically important anti-inflammatory effects in man are further suggested by trials demonstrating benefits of fatty acids in psoriasis, among others. ${ }^{21}$

After 8 weeks of treatment, there was a clear improvement in all the study endpoints in the group with Oravex ${ }^{\circledR}$ added to

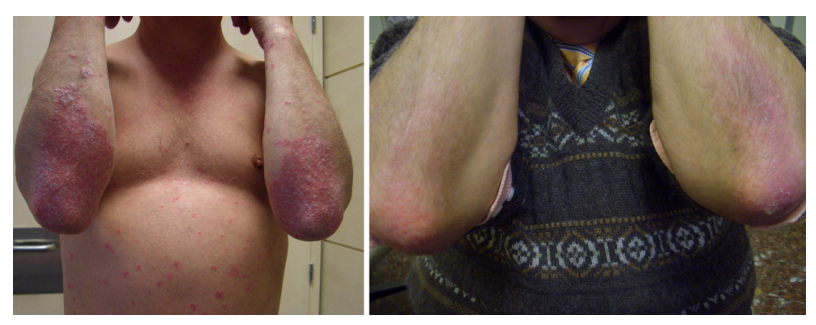

Figure 4 Patient I: reduction in erythema, desquamation and infiltration after the combined treatment with Oravex ${ }^{\circledR}$ and tacalcitol.

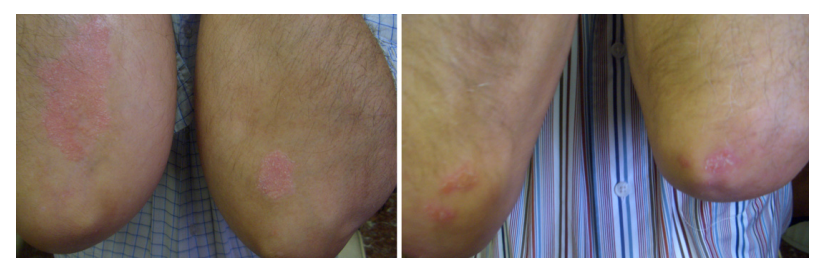

Figure 5 Patient 2: reduction in desquamation and infiltration after the combined treatment with Oravex ${ }^{\circledR}$ and tacalcitol.

the treatment with tacalcitol versus the control group, treated exclusively with tacalcitol.

Despite the low number of patients recruited to the study, the statistical significance of the differences between groups clearly indicates that a global improvement is achieved with the addition of Oravex ${ }^{\circledR}$ to the treatment with tacalcitol.

\section{Conclusion}

Supplementary treatment with omega-3 fatty acids complements topical treatment in psoriasis, and makes a significant contribution to reducing PASI and NAPSI, and improving DLQI; and in reducing scalp lesion and pruritus, erythema, scaling, and infiltration of the treated areas.

\section{Disclosure}

The authors declare no conflicts of interest.

\section{References}

1. Ferrándiz C. Dermatosis eritematoescamosas (I). Psoriasis. Eritrodermias. En: Ferrándiz C, editor. Dermatología Clínica. Madrid: Mosby; 1996:149-156.

2. Casanova JM, Martí RM. Inmunopatogénesis de la psoriasis. Piel. 1993;7:486-495.

3. Puig Sanz L. Psoriasis, a systemic disease? Actas Dermosifiliog. 2007; 98:396-402.

4. Love TJ, Qureshi AA, Karlson EW, Gelfand JM, Choi HK. Prevalence of the Metabolic Syndrome in Psoriasis: Results From the National Health and Nutrition Examination Survey, 2003-2006. Arch Dermatol. 2010 Dec 20. [Epub ahead of print].

5. Prey S, Paul C, Bronsard V, et al. Cardiovascular risk factors in patients with plaque psoriasis: a systematic review of epidemiological studies. J Eur Acad Dermatol Venereol. 2010;24 Suppl 2:23-30.

6. Mallbris L, Ritchin CT, Stahle M. Metabolic disorders in patients with psoriasis and psoriatic arthritis. Current Rheumatol Rev. 2006; 8(355):63.

7. Kagami S, Rizzo HL, Lee JJ, Koguchi Y, Blauvelt A. Circulating Th17, Th22, and Th1 cells are increased in psoriasis. J Invest Dermatol. 2010; 130:1373-1383.

8. Casanova JM. Diagnóstico y tratamiento de la psoriasis. Piel. 1987;2: 416-424.

9. De Pablo, Guerra A. Tratamiento de la psoriasis en pacientes ambulatorios. Piel. 1993;8:388-396.

10. Graaves MV, Weinstein GD. Treatment of psoriasis. $N$ Engl $J$ Med. 1995;332:581-587.

11. Kragballe K. Vitamin D3 analogues. Dermatol Clin. 1995;13: 835-839.

12. Lebwohl MG. The evolution of vitamin D analogues for the treatment of psoriasis. Arch Dermatol. 1995;131:1323-1324. 
13. Ohta T, Okabe K, Azuma Y, Kiyoki M. In vivo microautoradiography of tacalcitol following topical application to normal rats in vitro metabolism in human keratinocites. Arch Dermatol Res. 1996;288: 188-196.

14. Sato H, Sugimoto I, Matsunaga T, Tsuchimoto M, Ohta T, Uno H, et al. Tacalcitol inhibits phorbol esterinduced epidermal proliferation and cutaneous inflammation, and induces epidermal differenciation in mice. Arch Dermatol Res. 1996;288:656-663.

15. Scarpa C. Tacalcitol Pomada: Un tratamiento eficaz y bien tolerado de la psoriasis. J Eur Acad Dermatol Venereol. 1996;6:142-146.

16. Wolters M. Diet and psoriasis: experimental data and clinical evidence. Br J Dermatol. 2005;153:706-714

17. Wolters M. The significance of diet and associated factors in psoriasis. Hautartz. 2006;57:999-1004.
18. Kragballe K, Fogh K. A low-fat diet supplemented with dietary fish oil (Max-EPA) results in improvement of psoriasis and in formation of leukotriene B5. Acta Derm Venereol. 1989;69:23-28.

19. Kojima T, Terano T, Tanabe E, Okamoto S, Tamura Y, Yoshida S. Long-term administration of highly purified eicosapentaenoic acid provides improvement of psoriasis. Dermatologica. 1991;182: 225-230.

20. Lassus A, Dahlgren AL, Halpern MJ, Santalahti J, Happonen HP. Effects of dietary supplementation with polyunsaturated ethyl ester lipids (Angiosan) in patients with psoriasis and psoriatic arthritis. J Int Med Res. 1990;18:68-73.

21. Mori TA, Beilin LJ. Omega-3 fatty acids and inflammation. Curr Atheroscler Rep. 2004;6:461-467.

\section{Publish your work in this journal}

Clinical, Cosmetic and Investigational Dermatology is an international, peer-reviewed, open access, online journal that focuses on the latest clinical and experimental research in all aspects of skin disease and cosmetic interventions. All areas of dermatology will be covered; contributions will be welcomed from all clinicians and basic science researchers globally. This journal is indexed on CAS. The manuscript management system is completely online and includes a very quick and fair peer-review system, which is all easy to use. Visit http://www.dovepress.com/testimonials.php to read real quotes from published authors.

Submit your manuscript here: http://www.dovepress.com/clinical-cosmetic-and-investigational-dermatology-journal 